\title{
A Histogram based Hybrid Approach for Medical Image Denoising using Wavelet and Curvelet Transforms
}

\author{
K.S.Tamilselvan, \\ Assistant Professor(SGR), \\ Department of ECE, \\ Velalar College of \\ Engineering \&Technology, \\ Anna University, India
}

\author{
G.Murugesan,Ph.D \\ Professor \& Head, \\ Department of ECE, \\ Kongu Engineering College, \\ Anna University, India
}

\author{
Maj.(Retd).M.Vinothsaravanan,Ph. \\ D \\ Radiologist, \\ Jansons MRI Diagnostic(PVT) LTD, \\ Erode, India
}

\begin{abstract}
Medical images are analyzed for the diagnosis of various diseases like cancer, tumor and fracture etc... But, they are susceptible to different types of noises called as Gaussian noise, Speckle noise, Uniform noise, Impulse noise, etc...Therefore it is an important task to remove the noise from medical images especially in MRI,CT, PET,SPECT, Digital Mammogram and Ultrasound images. Selection of appropriate filter is a tough task. In this paper, we propose a technique that uses Wavelet Transform and Curvelet Transform for denoising the medical images based on the Histogram equalization.
\end{abstract}

\section{Key Words}

Medical images, Speckle noise, Impulse noise, MRI, CT, PET, SPECT, Digital Mammogram, Ultrasound images, Wavelet Transform, Curvelet Transform and Histogram equalization

\section{INTRODUCTION}

Image processing is an important step in clinical image diagnosis. Medical images are acquired and analyzed to identify the occurrence of abnormalities like tumor, fracture and blocks. Most of the medical images are affected by different types of noises during acquisition, storage and transmission. So denoising of an image is a basic step in medical image processing. The normally used filtering techniques are, median and mean filters[2] .But, a single smoothening or median filter is not enough to remove the noise completely.

\section{TYPES OF NOISES IN MEDICAL IMAGES}

\subsection{Gaussian Noise:}

Gaussian noise has a Gaussian distribution, with a bell shaped probability distribution function and given by,

$\mathrm{F}(\mathrm{g})=\frac{1}{\sqrt{2 \pi \sigma^{2}}} e^{-(g-m)^{2}} / 2 \sigma^{2}$

where $g$ represents the gray level, $m$ is the mean or average of the function, and $\sigma$ is the standard deviation of the noise[1].

\subsection{Salt and Pepper Noise:}

Salt and pepper noise is an impulse type of noise, and caused due to errors in data transmission. It has only two possible values, low and high. The corrupted pixels are set alternatively to the minimum or to the maximum value, giving the image a "salt and pepper" like appearance[3]. Unaffected pixels remain unchanged. For an 8-bit image, the typical value for pepper noise is 0 and for salt noise is 255 .

\subsection{Speckle Noise:}

Speckle noise is a repetitive type of noise and occurs in imaging systems such as laser and SAR (Synthetic Aperture Radar) . The source of this noise is attributed to random interference between the coherent returns[7]. The mathematical expression for this noise is given by,

$\mathrm{F}(\mathrm{g})=\frac{g^{\alpha-1}}{(\alpha-1) ! a^{\alpha}} e^{-\frac{g}{a}} \quad$ where $a^{\alpha}$ is variance and $g$ is the gray level.

\section{EXISTING METHODS AND THEIR LIMITATIONS}

Filters are the main components in the image restoration process. The main idea behind the restoration technique is convolution. It becomes a moving window operation with different types of filters used.

\subsection{Linear Filtering}

- Mean Filter

- LMS Adaptive Filter

\subsection{Nonlinearfiltering approach:}

- Median filter

\section{PROPOSED METHOD}

A hybrid technique of image denoising was proposed in this paper.. With the observation of advantage and disadvantages of different transforms, a combination of wavelet transform and curvelet transform was adapted to denoise the medical image. Histogram equalization technique is also used.

\subsection{Basic Denoising Concept}

Regarding medical images, noise and its characteristics are known earlier. Let the image $s(\mathrm{a}, \mathrm{b})$ is affected by a linear operation and noise $n(\mathrm{a}, \mathrm{b})$ is added to form the degraded image $w(\mathrm{a}, \mathrm{b})$. This is convolved with the restoration procedure $g(\mathrm{a}, \mathrm{b})$ to produce the restored image $\mathrm{y}(\mathrm{a}, \mathrm{b})$. 


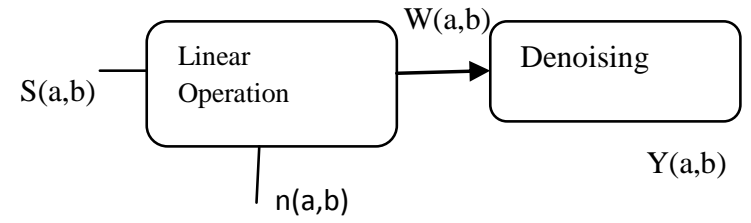

Figure 1. Basic Denoising concept

The "Linear operation" shown in Figure. 1 is the addition or multiplication of the noise $n(\mathrm{a}, \mathrm{b})$ to the signal $s(\mathrm{a}, \mathrm{b})$. Once the corrupted image $w(\mathrm{a}, \mathrm{b})$ is obtained, it is subjected to the denoising technique to get the denoised image $z(\mathrm{a}, \mathrm{b})$. The point of focus in this paper is comparing and contrasting several denoising techniques. Three popular techniques are discussed in this paper.

Denoising by wavelets in combination with Curevlet based technique is an advanced approach in noise reduction. Wavelet techniques consider thresholding while curvelet technique analysis is based on representation of the edges and singularities more efficiently and thus by removing the noise contents in an image.MRI images are mostly corrupted by random noise during the image acquisition time[5]. Such a noise introduces uncertainties in the measurement of quantitative parameters which disturbs the estimation of the different features of the analyzed tissues

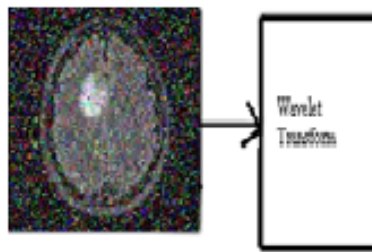

Input Image
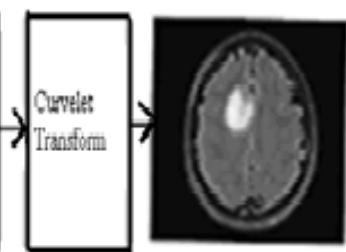

Output Image
Figure..2.Flow Diagram of Proposed method.

\subsection{Histogram Equalization}

\subsubsection{Steps in Histogram Equalization}

Step.1:For a given image A, we will now design a special point function $g e A(l)$ which is called the histogram.

Step.2: Equalizing point function for A. If $B(i ; j)=g_{e} A(A(i ; j))$, then our aim is to make $h B(l)$ as uniform/flat as possible irrespective of $h A(l)^{2}$

Step.3:Compare images by "mapping” their histograms into a standard histogram and sometimes "undo" the effects of some unknown processing. The techniques we are going to use to get $g_{e} A(l)$ are also applicable in histogram modification/specification.

Step.4:Stretch/Compress an image such that: Pixel values that occur frequently in A occupy a bigger dynamic range in $\mathrm{B}$, i.e., get stretched and become more visible.

\subsection{Noise reduction using Wavelet Transform}

\subsubsection{Concept of Wavelet Transform}

Image enhancement functions can be implemented independently from the wavelet filters and easily incorporated into the filter bank framework. De-noising can be viewed as an estimation problem to recover a true signal component $X$ from an observation $\mathrm{Y}$ where the signal component has been degraded by a noise signal $\mathrm{N}$.

$\mathrm{Y}=\mathrm{X}+\mathrm{N}$

The estimation is computed with a thresholding estimator in an orthonormal basis $\mathrm{B}=\{\mathrm{gm}\} 0<\mathrm{m}<\mathrm{N}$ as

$\mathrm{X}=\sum_{m=0}^{N-1} \sigma \mathrm{m}(\langle X, g m\rangle) g m$

where $\sigma_{\mathrm{m}}$ is a thresholding function that aims at eliminating noise components in the transform domain while preserving the true signal coefficients[1,8]. If the function $\sigma \mathrm{m}$ is modified to rather preserve or increase coefficient values in the transform domain, it is possible to enhance some features of interest in the true signal.

\subsubsection{Selection of Threshold Value}

Given the basic framework of de-noising using wavelet thresholding, it is clear that the threshold level parameter $T$ plays an essential role. Values too small cannot effectively get rid of noise component, while values too large will eliminate useful signal components. There are a variety of ways to determine the threshold value $T$ as discussed in this section.

\subsubsection{Thresholding operators for de-noising}

As a general rule, wavelet coefficients with larger magnitude are correlated with salient features in the image data. In that context, de-noising can be achieved by applying a thresholding operator to the wavelet coefficients (in the transform domain) followed by reconstruction of the signal to the original image (spatial) domain.

Typical threshold operators for de-noising include

Hard thresholding:

$\sigma_{\mathrm{T}}(\mathrm{x})=\left\{\begin{array}{l}x, \text { if }|x|>T \\ 0, \text { if }|x| \leq T\end{array}\right.$

Soft thresholding (wavelet shrinkage) :

$\sigma_{\mathrm{T}}(\mathrm{x})=\left\{\begin{aligned} x, \text { if }|x| & \geq T, \\ x+T, \text { if } x & \leq-T, \\ 0, \text { if }|x| & <T .\end{aligned}\right.$

Affine(firm) thresholding:

$$
\sigma_{\mathrm{T}}(\mathrm{x})=\left\{\begin{array}{c}
x-T, \text { if } x \geq T, \\
2 x+T, \text { if }-T \leq x \leq-T / 2, \\
2 x-T, \text { if } T / 2 \leq x \leq T \\
0, \text { if }|x|<T .
\end{array}\right.
$$


The threshold value ' $\mathrm{T}$ ' which changes across wavelet scales and spatial locations, can be classified as,

Global Threshold: a single value $T$ is to be applied globally to all empirical wavelet coefficients at different scales, $T=$ constant.

Levl-Depeendent Threshold: a different threshold value $T$ is selected for each wavelet analysis level (scale).T=T(j), $j=1,2,3, \ldots J . J$ is the coarsest level for wavelet expansion to be processed.

\section{5 .EXPERIMENTAL RESULTS}

\subsection{Concept of Curvelet Transform}

Curvelet transform is a new multi-scale representation and it is most suitable for the objects with curves. It represents the edges and singularities more efficiently[10]. It is a new extension to the wavelet transform and ridgelet transform in two dimensional images which aims to deal with interesting phenomena occurring along the curves. It differs from other transforms in that degree of localization. It is designed to handle curves using only a small number of coefficients. Hence the

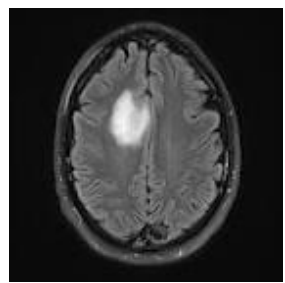

Original Input image

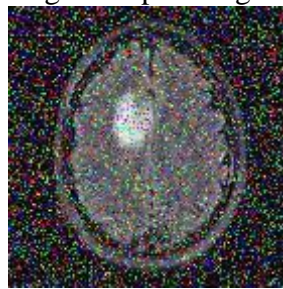

Input Image with Speckle Noise

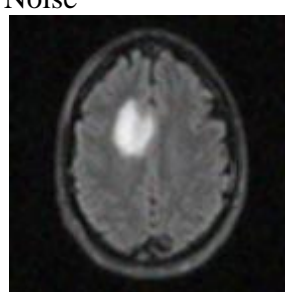

Input Image with Uniform noise Filtered

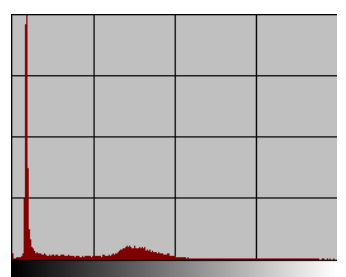

Histogram of Input Image

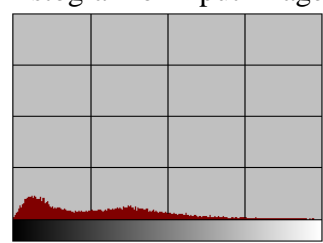

Histogram of Input Image with Speckle Noise

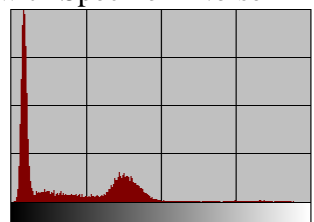

Histogram of Uniform

Noise Filtered

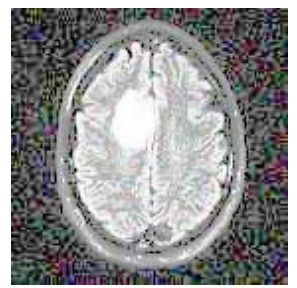

Input Image with Gaussian noise

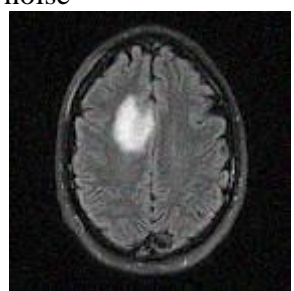

Image with Histogram

Equalization

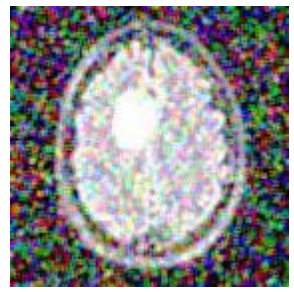

Image with Histogram

Equalization

curvelet transforms handle the curve discontinues well. It is a high-dimensional generalization of the wavelet transform designed to represent images at different scales and different orientations[11].Because of these properties, it is used for extracting the important features from the medical images and segmentation accurately. It is proven to be effective in the detection of image activity along curves instead of radial directions which are the most comprising objects of medical images.To fix the curvelet for a suitable application, it is worthful to analyze the curvelets by applying parabolic dilations, rotations, and translations to a specifically shaped function $\psi$; they are indexed by a scale parameter $a(0<a<1)$, a location $b$, and an orientation $\vartheta$ and are nearly of the form,

$$
\Psi \text { a,b, } \theta(\mathrm{x})=\mathrm{a}^{-3 / 4} \Psi(\operatorname{DaR} \theta(\mathrm{x}-\mathrm{b})),
$$

$\mathrm{D}_{\mathrm{a}}=\left(\begin{array}{cc}1 / a & 0 \\ 0 & 1 / \sqrt{a}\end{array}\right)$ Here, $\mathrm{Da}$ is a parabolic scaling matrix, $\mathrm{R} \theta$ is a rotation by $\theta$ radians.

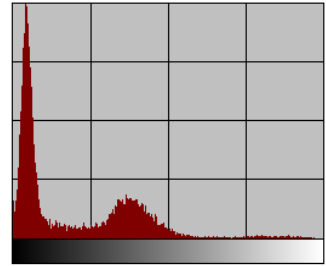

Histogram of Input Image with Gaussian Noise

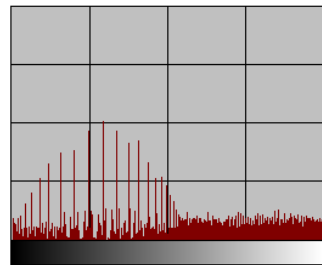

Histogram of Histogram Equalized Image

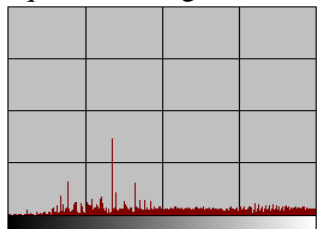

Histogram of equalized Image

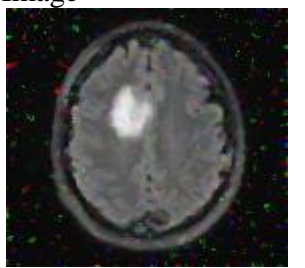

Curvelet Filtered image

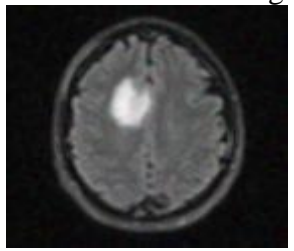

Image with Gaussian Noise Filtered

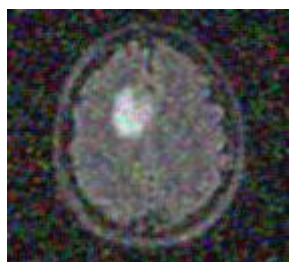

Input Image with Uniform noise

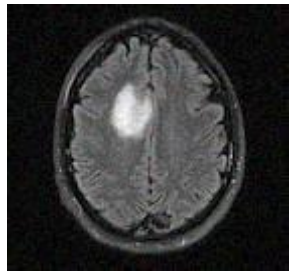

Image with Speckle Noise Filtered

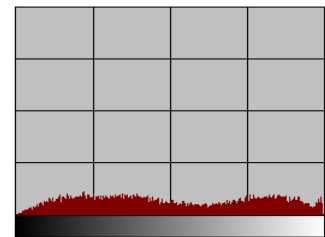

Histogram Equalized Image

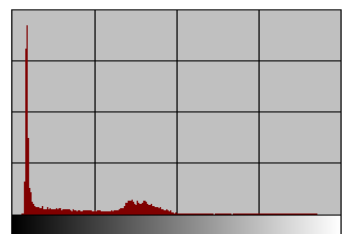

Histogram of Curvelet Filtered Image

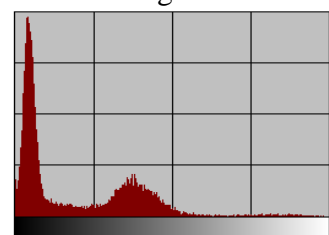

Histogram of Filtered Image 


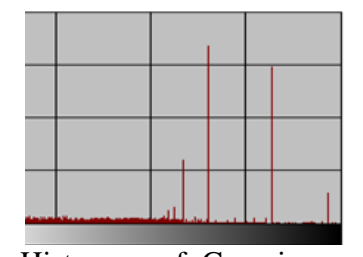

Histogram of Gaussian Noise Filtered

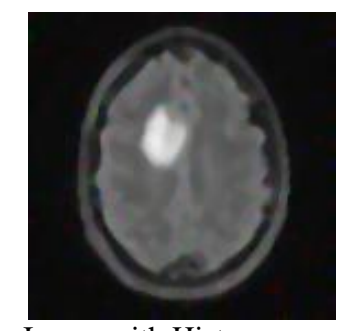

Image with Histogram Equalization

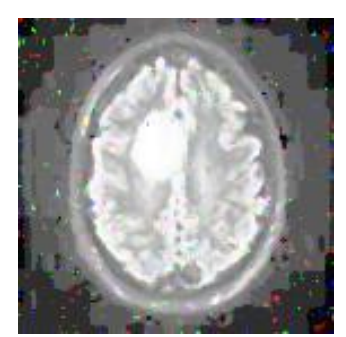

Equalized Image

Wavelet Filtered Image

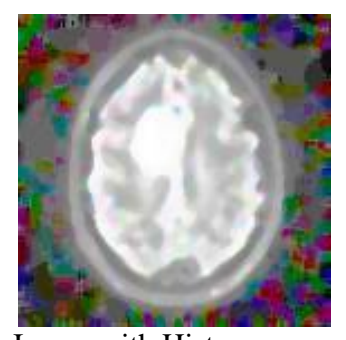

Image with Histogram Equalization

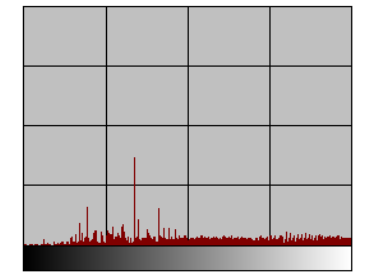

Histogram equalized Image

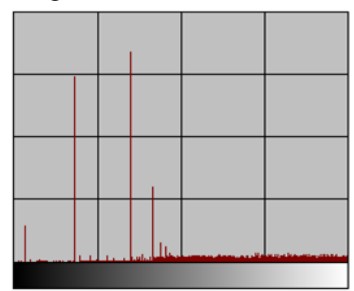

Histogram of Wavelet Filtered Image

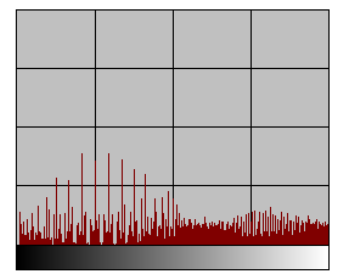

Histogram of proposed method

\section{Figure.3.Histogram of images before and after denoising}

\section{COMPARISON OF RESULTS AND DISCUSSION}

Table..1.Comparison of SNR, STD Deviation, Median and Mean of various Denoising Techniques

\begin{tabular}{|l|c|c|c|c|c|c|c|}
\hline \multirow{2}{*}{ Technique } & \multicolumn{2}{|c|}{ SNR } & \multicolumn{2}{c|}{ Standard Deviation } & \multicolumn{2}{c|}{ Mean } \\
\cline { 2 - 8 } & Filtered & 'H' Equalized & Filtered & 'H' Equalized & Filtered & 'H' Equalized & \\
\hline Wiener Filter & 1.727 & 2.7374 & $3.7 \mathrm{e}+002$ & $3.7 \mathrm{e}+002$ & 28 & 123 & 77 \\
\hline $\begin{array}{l}\text { Curvelet } \\
\text { Technique }\end{array}$ & 3.1420 & 3.9816 & $2.8 \mathrm{e}+002$ & $2.8 \mathrm{e}+002$ & 25 & 125 & 77 \\
\hline Wavelet Technique & 4.1959 & 4.8905 & $3 \mathrm{e}+002$ & $3 \mathrm{e}+002$ & 21 & 126 & 77 \\
\hline Proposed Method & $\mathbf{4 . 4 6 6 9}$ & $\mathbf{5 . 5 4 3 3}$ & $\mathbf{2 . 1 e + 0 0 2}$ & $\mathbf{2 . 1 e + 0 0 2}$ & $\mathbf{2 2}$ & $\mathbf{1 2 9}$ & $\mathbf{7 7}$ \\
\hline
\end{tabular}

Table.2. Comparison of Histogram Statistics of various Denoising Techniques

\begin{tabular}{|l|c|c|c|c|c|c|c|c|}
\hline \multirow{2}{*}{ Technique } & \multicolumn{2}{|c|}{ 'H' Maximum } & \multicolumn{2}{|c|}{ No. of Zeros } & \multicolumn{2}{|c|}{ No. of Non-zeros } & \multicolumn{2}{c|}{ Mode } \\
\cline { 2 - 10 } & Filtered & 'H' Equalized & Filtered & 'H' Equalized & Filtered & 'H' Equalized & Filtered & 'H' Equalized \\
\hline Wiener Filter & $6 \mathrm{e}+003$ & $6.3 \mathrm{e}+003$ & 0 & 203 & 256 & 53 & 255 & 255 \\
\hline $\begin{array}{l}\text { Curvelet } \\
\text { Technique }\end{array}$ & $3.2 \mathrm{e}+003$ & $3.5 \mathrm{e}+003$ & 31 & 144 & 225 & 112 & 11 & 82 \\
\hline $\begin{array}{l}\text { Wavelet } \\
\text { Technique }\end{array}$ & $3 \mathrm{e}+003$ & $3.4 \mathrm{e}+003$ & 30 & 149 & 226 & 107 & 11 & 89 \\
\hline $\begin{array}{l}\text { Proposed } \\
\text { Method }\end{array}$ & $\mathbf{2 . 2 e + 0 0 3}$ & $\mathbf{2 . 5 e + 0 0 3}$ & $\mathbf{2 8}$ & $\mathbf{1 5 6}$ & $\mathbf{2 2 8}$ & $\mathbf{1 0 0}$ & $\mathbf{1 1}$ & $\mathbf{8 6}$ \\
\hline
\end{tabular}

Table.3. Comparison of Histogram Statistics of various types of Noises and Filtering Techniques

\begin{tabular}{|c|l|c|c|c|c|c|c|c|}
\hline $\begin{array}{c}\text { Type of } \\
\text { Noise }\end{array}$ & Type of Image & $\begin{array}{c}\text { Standard } \\
\text { Deviation }\end{array}$ & $\begin{array}{c}\text { 'H' } \\
\text { Maximum }\end{array}$ & Median & $\begin{array}{c}\text { No. of } \\
\text { Zeros }\end{array}$ & $\begin{array}{c}\text { No. of Non- } \\
\text { Zeros }\end{array}$ & $\begin{array}{c}\text { Mode } \\
\text { Processing Time } \\
(\mathrm{mS})\end{array}$ \\
\hline \multirow{3}{*}{$\begin{array}{c}\text { Gaussian } \\
\text { Noise }\end{array}$} & Noisy Image & $1.5 \mathrm{e}+002$ & $3.4 \mathrm{e}+002$ & 19 & 11 & 245 & 11 & 14 \\
\cline { 2 - 10 } & Filtered Image & $2 \mathrm{e}+002$ & $1.5 \mathrm{e}+002$ & 19 & 16 & 240 & 11 & 14 \\
\cline { 2 - 10 } & $\begin{array}{l}\text { 'H' Equalized } \\
\text { Image }\end{array}$ & $\mathbf{2 0}$ & $\mathbf{2 . 6 e + 0 0 2}$ & $\mathbf{1 3 0}$ & $\mathbf{0}$ & $\mathbf{2 5 6}$ & $\mathbf{0}$ & $\mathbf{1 6}$ \\
\hline \multirow{3}{*}{$\begin{array}{c}\text { Speckle } \\
\text { Noise }\end{array}$} & Noisy Image & $1.5 \mathrm{e}+002$ & $2.3 \mathrm{e}+002$ & 66 & 3 & 253 & 0 & 14 \\
\cline { 2 - 10 } & Filtered Image & 82 & $2.1 \mathrm{e}+002$ & 60 & 30 & 226 & 46 & 14 \\
\cline { 2 - 10 } & $\begin{array}{l}\text { 'H' Equalized } \\
\text { Image }\end{array}$ & $\mathbf{5 7}$ & $\mathbf{2 . 8 e + 0 0 2}$ & $\mathbf{1 3 3}$ & $\mathbf{0}$ & $\mathbf{2 5 6}$ & $\mathbf{3}$ & $\mathbf{3 2}$ \\
\hline \multirow{3}{*}{$\begin{array}{c}\text { Uniform } \\
\text { Noise }\end{array}$} & Noisy Image & $1.5 \mathrm{e}+002$ & $1.9 \mathrm{e}+002$ & 19 & 10 & 246 & 12 & 16 \\
\cline { 2 - 10 } & Filtered Image & $1.9 \mathrm{e}+002$ & $1.5 \mathrm{e}+002$ & 19 & 17 & 239 & 11 & 16 \\
\cline { 2 - 9 } & $\begin{array}{l}\text { 'H' Equalized } \\
\text { Image }\end{array}$ & $\mathbf{2 0}$ & $\mathbf{2 . 6 e + 0 0 2}$ & $\mathbf{1 3 0}$ & $\mathbf{0}$ & $\mathbf{2 5 6}$ & $\mathbf{0}$ & $\mathbf{4 6}$ \\
\hline
\end{tabular}


Two types of comparisons are made from the results. One is comparison of SNR, STD Deviation, Median and Mean for various techniques of Image Denoising. From the above observations it is clear that most of the features are best in the proposed method compared with other techniques. Second comparison is between type of noise and its Histogram characteristics. From this it is observed that for some noise types, filtered image is better than the Histogram equalized image. This depends on the type of irrespective of the image.

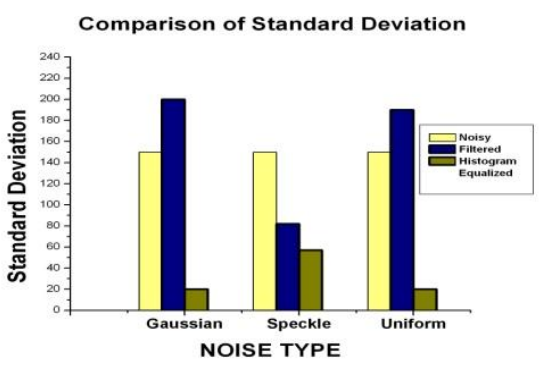

Figure .4.Comparison of STD Deviation

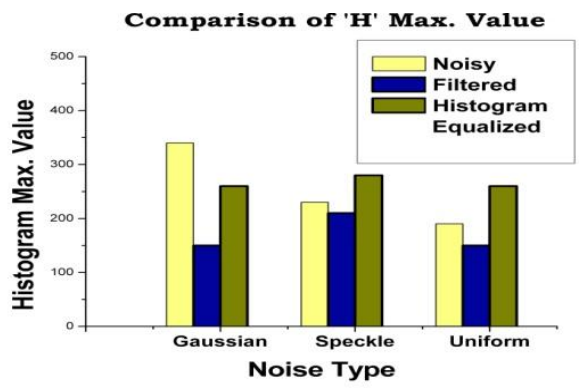

Figure .5.Comparison of 'H' Maximum

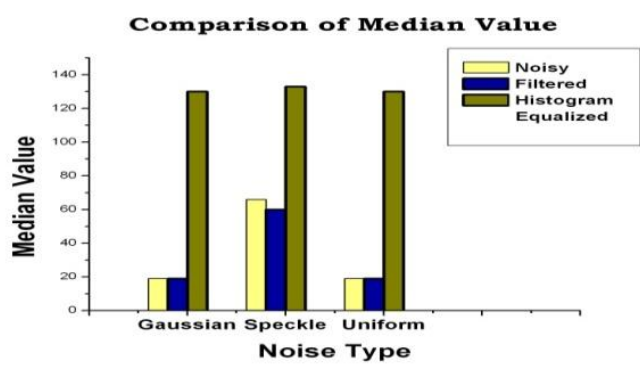

Figure 6.Comparison of Median Values

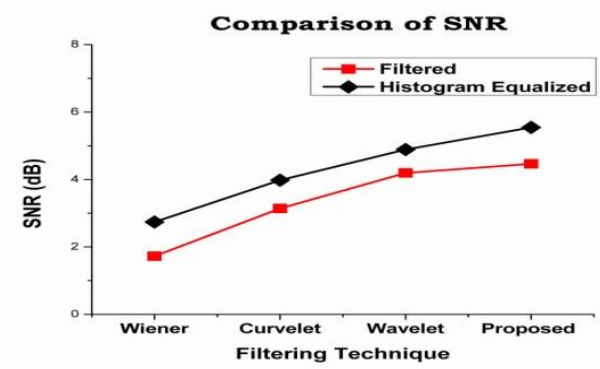

Figure .7.Comparison of SNR

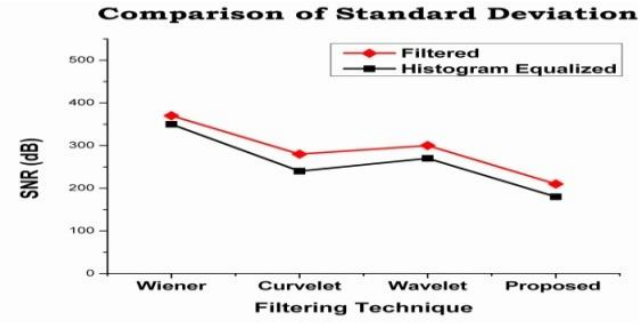

Figure.8. Comparison of STD Deviation

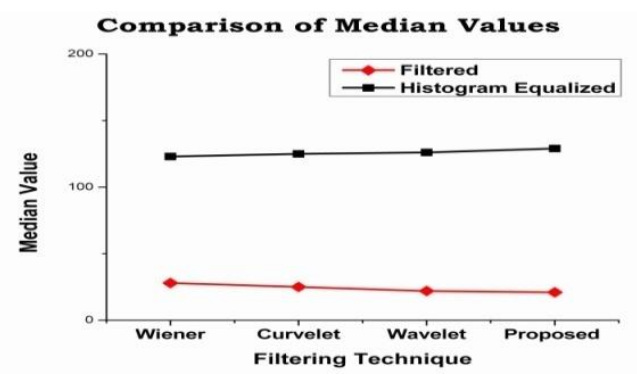

Figure 9.Comparison of Median values

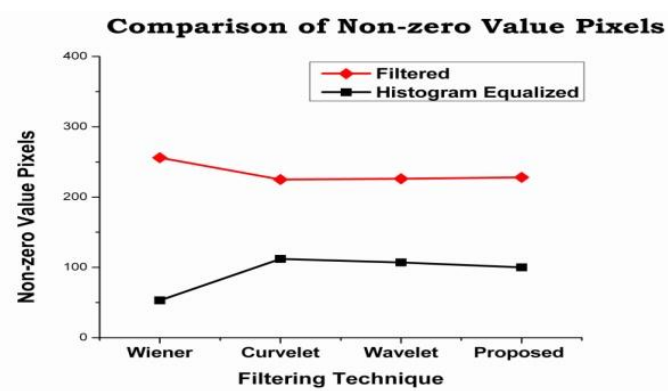

Figure .10. Comparison of Non-zero value pixels

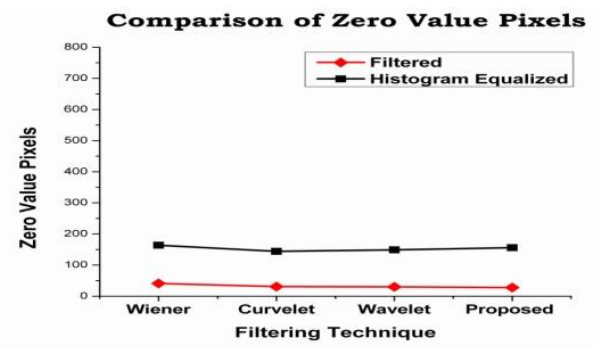

Figure .11. Comparison of zero value pixels

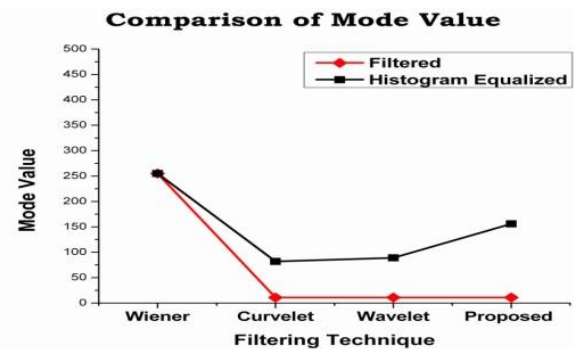

Figure .12. Comparison of different Mode Values 


\section{CONCLUSION}

A new hybrid technique for the removal of Gaussian, Uniform and Speckle noises from Medical Images. New technique followed was based on the combination of Curvelet and Wavelet Transform. We also utilize the Histogram equalization to smoothen the noisy image. It is found to be more suitable and more efficient than the existing methods of image denoising particularly for the removal of above mentioned three types of noises. Various results were obtained and analyzed by comparing with each other techniques in terms of Signal to Noise Ratio, Standard Deviation, Mean and Median for MRI image. In future we decide to extend this technique to the noise reduction of all types of medical images like, CT, PET, SPECT, FMRI and X-Ray images.

\section{REFERENCES}

[1]. Ashok Saini, "Reduction of Noise from Enhanced Image Using Wavelets",International Journal of Electronics Engineering, 3 (2), 2011, pp. 275-277,"

[2].Sandeep Kumar, Puneet Verma, "Comparison of Different Enhanced Image Denoising with Multiple Histogram Techniques", International Journal of Soft Computing and Engineering (IJSCE) ISSN: 2231-2307, Volume-2, Issue2, May 2012.

[3]. S.Satheesh, Dr.KVSVR Prasad,"Medical Image Denoising using Adaptive Threshold based on Contourlet Transform", Advanced Computing: An International Journal ( ACIJ ), Vol.2, No.2, March 2011

[4]. J. Fowler, "The redundant discrete wavelet transform and additive noise," IEEE Signal Processing Letters, vol. 12, no. 9, pp. 629-632,2005
[5]. L. Parthiban and R. Subramanian, "MRI image denoising for telemedicine," 8th Int. Conf. on e-Health Networking,Applications and Services 2006 (HEALTHCOM 2006), pp. 188- 191, 17-19 Aug. 2006.

[6]. M. Abdullah-Al-Wadud, Md. Hasanul Kabir, M. Ali Akber Dewan, and Oksam Chae, "A dynamic histogram equalization for image contrast enhancement", IEEE Transactions. Consumer Electron., vol. 53, no. 2, pp. 593600, May 2007

[7]. Sudha, G.R.Suresh, and R. Sukanesh , "Speckle Noise Reduction in Ultrasound Images by Wavelet Thresholding based on Weighted Variance", International Journal of Computer Theory and Engineering, Vol.1, No.1, April 2009, 1793-8201

[8].Pei-yan FEI Bao-long GUO," Image Denoising based on the Dyadic Wavelet Transform," Proceedings of the Fifth International Conference on Computational Intelligence and Multimedia Applications (ICCIMA'03) 0-7695-19571/03 2003 IEEE

[9]. Eric I. Balster and Yuan E Zheng, "Fast, Feature-Based Wavelet Shrinkage Algorithm for Image Denoising", KIMAS 2003. October 1-3,2003,B oston. MA,USA Copyright 0-7803-7958-6/03/\$17.0B0 2003 IEEE

[10]. Jianwei Ma and Gerlind Plonka, "The Curvelet Transform-A review of recent applications",IEEE Signal Processing Magazine [120] March 2010

[11]. E. J. CANDÈS and L. DEMANET, "Curvelets and Fourier integral operators", C. R. Math. Acad. Sci. Paris 336

(2003),

395-398 